\begin{tabular}{|c|c|}
\hline Title & A n efficient finite element analysis of magnetooptic channel waveguides \\
\hline Author(s) & Koshiba, M.; Zhuang, X .P. \\
\hline Citation & $\begin{array}{l}\text { Journal of Lightwave Technology, 11(9), 1453-1458 } \\
\text { https://doi.org/10.1109/50.241935 }\end{array}$ \\
\hline Issue Date & $1993-09$ \\
\hline Doc URL & http:/hdl .handle.net/2115/6039 \\
\hline Rights & $\begin{array}{l}\text { (1993 IEEE. Personal use of this material is permitted. However, permission to reprint/republish this material for } \\
\text { advertising or promotional purposes or for creating new collective works for resale or redistribution to servers or lists, } \\
\text { or to reuse any copyrighted component of this work in other works must be obtained from the IEEE. } \\
\text { IEEE, Journal of Lightwave Technology, 11(9), 1993, p1453-1458 }\end{array}$ \\
\hline Type & article \\
\hline File Information & JLT11_9.pdf \\
\hline
\end{tabular}

Instructions for use 


\title{
An Efficient Finite-Element Analysis of Magnetooptic Channel Waveguides
}

\author{
Masanori Koshiba, Senior Member, IEEE, and Xiu-Ping Zhuang
}

\begin{abstract}
A finite-element method based on the scalar-wave approximation is developed for the analysis of magnetooptic waveguides. A simple and efficient iterative method is proposed for solving a nonlinear eigenvalue equation derived from the scalar finite-element approach. To show the validity and usefulness of this method, examples are computed for magnetooptic rib-type and ridge-type waveguides. Subsequently, we discuss the waveguide structures which have larger nonreciprocal phase shift.
\end{abstract}

\section{INTRODUCTION}

A MAGNETOOPTIC waveguide is one of the key elements in nonreciprocal devices such as isolators and circulators. Theoretical studies on the nonreciprocity of magnetooptic waveguides have mainly focused on planar (two-dimensional) waveguides [1]-[4]. Two-dimensional waveguides can trap optical fields in the direction of the thickness ( $y$ direction), but allow the fields to spread in the horizontal direction ( $x$ direction). In order to facilitate the construction of integrated nonreciprocal devices, channel (three-dimensional) waveguides, which trap optical fields in both $x$ and $y$ directions, are more important. It is, in general, difficult to analyze three-dimensional waveguides with nonreciprocal properties, and approximate analytical methods, such as the Marcatili method [5] and the effective index method have been used [6]-[8].

In this paper, a new numerical solution method, which is more accurate and can be applied to various magnetooptic channel waveguides, is developed. This approach is based on the finite-element method and the scalar-wave approximation [9]-[11]. A simple and efficient iterative method is proposed for solving a nonlinear eigenvalue equation derived from the scalar finite-element approach. The validity and usefulness of this method are confirmed by analyzing the magnetooptic ribtype and ridge-type waveguides. We also discuss possible ways to get larger nonreciprocal phase shift. Because the formulation is based on the scalar-wave approximation, spurious solutions that are included in the vector finite-element method [12] do not appear.

Manuscript received August 14, 1992; revised February 5, 1993. This work was partially supported by Grant-In-Aid for Scientific Research on Priority Area, Ultrafast and Ultra-Parallel Optoelectronics from the Ministry of Education, Science and Culture of Japanese Government.

The authors are with the Department of Electronic Engineering, Hokkaido University, Sapporo, 060 Japan.

\section{BASIC EQUATIONS}

With a time dependence of the form $\exp (j \omega t)$ being implied, Maxwell's equations are

$$
\begin{aligned}
\nabla \times \boldsymbol{E} & =-j \omega \mu_{0} \boldsymbol{H} \\
\nabla \times \boldsymbol{H} & =j \omega \epsilon_{0}\left[\epsilon_{r}\right] \boldsymbol{E} \\
\nabla \cdot \boldsymbol{H} & =0 \\
\nabla \cdot\left(\left[\epsilon_{r}\right] \boldsymbol{E}\right) & =0
\end{aligned}
$$

where $\omega$ is the angular frequency, $\boldsymbol{E}$ and $\boldsymbol{H}$ are the electric and magnetic fields, respectively, $\epsilon_{0}$ and $\mu_{0}$ are the permittivity and permeability of free space, respectively, and $\left[\epsilon_{r}\right]$ is the relative permittivity tensor.

We consider magnetooptic channel waveguides as shown in Fig. 1, where all the materials are assumed to be lossless, light propagates along the $z$ direction, and the dc magnetic field is applied in the $x$ direction. The relative permittivity tensor of the magnetooptic material can be written as

$$
\left[\epsilon_{r}\right]=\left[\begin{array}{ccc}
n_{x}^{2} & 0 & 0 \\
0 & n_{y}^{2} & j \delta \\
0 & -j \delta & n_{z}^{2}
\end{array}\right]
$$

where $n_{x}, n_{y}$, and $n_{z}$ are the refractive indexes in the $x, y$, and $z$ directions, respectively, and $\delta$ represents the first-order magnetooptic effect, which causes nonreciprocal nature and is related to the Faraday rotation.

Rewriting (1)-(4) in component form, we have

$$
\begin{gathered}
\frac{\partial E_{z}}{\partial y}+j \nu \beta E_{y}=-j \omega \mu_{0} H_{x} \\
-j \nu \beta E_{x}-\frac{\partial E_{z}}{\partial x}=-j \omega \mu_{0} H_{y} \\
\frac{\partial E_{y}}{\partial x}-\frac{\partial E_{x}}{\partial y}=-j \omega \mu_{0} H_{z} \\
\frac{\partial H_{z}}{\partial y}+j \nu \beta H_{y}=j \omega \epsilon_{0} n_{x}^{2} E_{x} \\
-j \nu \beta H_{x}-\frac{\partial H_{z}}{\partial x}=j \omega \epsilon_{0} n_{y}^{2} E_{y}-\omega \epsilon_{0} \delta E_{z} \\
\frac{\partial H_{y}}{\partial x}-\frac{\partial H_{x}}{\partial y}=\omega \epsilon_{0} \delta E_{y}+j \omega \epsilon_{0} n_{z}^{2} E_{z}
\end{gathered}
$$


where $\left\{E_{x}\right\}$ is the global electric field vector, $\{0\}$ is a null vector, $n_{\text {eff }}=\beta / k_{0}$ is the effective refractive index, and the submatrices of $[K]$ and $[M]$ are given by

$$
\begin{gathered}
{[K]=\sum_{e} \iint_{e}\left[n_{x}^{2}\{N\}\{N\}^{T}-\frac{n_{x}^{2}}{n_{z}^{2}} \frac{\partial\{N\}}{\partial \bar{x}} \frac{\partial\{N\}^{T}}{\partial \bar{x}}\right.} \\
\left.-\frac{\partial\{N\}}{\partial \bar{y}} \frac{\partial\{N\}^{T}}{\partial \bar{y}}\right] d \bar{x} d \bar{y} \\
{[M]=\sum_{e} \iint_{e}\{N\}\{N\}^{T} d \bar{x} d \bar{y}}
\end{gathered}
$$

Here $\bar{x}=k_{0} x, \bar{y}=k_{0} y$, and the summation $\sum_{e}$ extends over all different elements.

Similarly, substituting (25) into (23), and using the variational principle, we obtain the following eigenvalue equation for the $E^{y}$ modes:

$$
\left[K\left(n_{\mathrm{eff}}\right)\right]\{H\}-n_{\mathrm{eff}}^{2}[M]\left\{H_{x}\right\}=\{0\}
$$

with

$$
\begin{aligned}
{\left[K\left(n_{\mathrm{eff}}\right)\right]=} & \sum_{e} \iint_{e}\left[\{N\}\{N\}^{T}-\frac{n_{z}^{2}}{\sigma} \frac{\partial\{N\}}{\partial \bar{x}} \frac{\partial\{N\}^{T}}{\partial \bar{x}}\right. \\
& -\frac{n_{y}^{2}}{\sigma} \frac{\partial\{N\}}{\partial \bar{y}} \frac{\partial\{N\}^{T}}{\partial \bar{y}} \\
& \left.+\nu n_{\mathrm{eff}} \frac{\delta}{\sigma}\left(\{N\} \frac{\partial\{N\}^{T}}{\partial \bar{y}}+\frac{\partial\{N\}}{\partial \bar{y}}\{N\}^{T}\right)\right] \\
& \cdot d \bar{x} d \bar{y} \\
{[M] } & =\sum_{e} \iint_{e} \frac{n_{z}^{2}}{\sigma}\{N\}\{N\}^{T} d \bar{x} d \bar{y} .
\end{aligned}
$$

\section{Method of Numerical Calculation}

We define the normalized nonreciprocal phase shift $\phi$ as follows [7], [8]:

$$
\phi=\left(\phi_{f}-\phi_{b}\right) / l
$$

where $l$ is a waveguide length, and the retardations $\phi_{f}$ and $\phi_{b}$, respectively, for the $+z$ and $-z$ propagations are given by

$$
\begin{aligned}
\phi_{f} & =\frac{2 \pi l}{\lambda}\left(n_{f x}-n_{f y}\right) \\
\phi_{b} & =\frac{2 \pi l}{\lambda}\left(n_{b x}-n_{b y}\right) .
\end{aligned}
$$

Here $n_{f x}, n_{f y}$ are the effective refractive indexes of the fundamental $E^{x}\left(E_{11}^{x}\right), E^{y}\left(E_{11}^{y}\right)$ modes in the case of $+z$ propagation, respectively, and $n_{b x}, n_{b y}$ are the effective refractive indexes of the $E_{11}^{x}, E_{11}^{y}$ modes in the case of $-z$ propagation, respectively.

Substituting (33) and (34) into (32) and noting that the effective refractive indexes of the $E_{11}^{x}$ mode propagating in the $+z$ and $-z$ directions are the same $\left(n_{f x}=n_{b x}\right)$, we obtain

$$
\phi=\frac{2 \pi}{\lambda}\left(n_{b y}-N_{f y}\right) .
$$

Although (26) for the $E^{x}$ modes is a linear generalized eigenvalue equation, (29) for the $E^{y}$ modes is a nonlinear generalized eigenvalue equation. Hence, we use the following iterative scheme.

(i) Specify $\lambda, n_{x}, n_{y}, n_{z}$, and $\delta$ as input data and calculate the coefficient matrix $[M]$. (ii) Assign initial value to $n_{\text {eff }}$ in an arbitrary way. A convenient way to choose this value is to use that for the $\delta=0$ case; we adopt this way in the present paper. (iii) Calculate the nonlinear coefficient matrix $\left[K\left(n_{\text {eff }}\right)\right]$. (iv) To obtain a new value of $n_{\text {eff }}$, solve the eigenvalue equation (29). (v) Iterate procedures (iii) and (iv) until the solution (eigenvalue, $n_{\text {eff }}$ ) converges within the desired criterion.

Fig. 2 shows the flowchart of the iterative process, where $\Delta$ is the value for judging the convergence. In this calculation we set $\Delta=10^{-10}$, and the convergent solution is obtained within four or five iterations.

\section{NUMERICAL RESULTS AND DISCUSSION}

We consider the magnetooptic channel waveguides as shown in Fig. 1 , where the wavelength $\lambda$ is $1.152 \mu \mathrm{m}$ and the refractive indexes of a substrate and a top layer are $n_{s}=1.95$ and $n_{c}=1.0$, respectively. The refractive index $n_{x}=n_{y}=$ $n_{z} \equiv n$ and the off-diagonal component of the relative permittivity tensor $\delta$ of magnetooptic materials are given in Table I [2], [7], [8], [13]. For simplicity, we assume the artificial boundary walls $x= \pm X / 2, y=-Y_{s}$, and $y=t+Y_{c}$ (magnetooptic rib waveguides) or $y=t+h+Y_{c}$ (magnetooptic ridge waveguides) far from the core region.

\section{A. Magnetooptic Rib Waveguides}

We consider the magnetooptic rib waveguides as shown in Fig. 1(a) and (b), where the rib width $W$ and rib height $d$ are $3 \mu \mathrm{m}$ and $12 \mathrm{~nm}$, respectively, $X=20.6 \mu \mathrm{m}, Y_{s}=1.9 \mu \mathrm{m}$, and $Y_{c}=0.5 \mu \mathrm{m}$. A magnetooptic material is used as a guided layer [7], [8] and a substrate [4], [14] in Fig. 1(a) and (b), respectively.

The magnitude of the nonreciprocal phase shift $|\phi|$ as a function of LaGa:YIG film thickness is shown in Fig. 3(a) by the solid line. The results obtained agree approximately with the experimental results [7]. The results of the planar waveguide $(d=0)$ are also shown in Fig. 3(a), by the dotted line. For larger values of $t$, the rib waveguide structure considered here is almost like the planar structure, and therefore the value of $|\phi|$ for the three-dimensional waveguide approaches that for the two-dimensional waveguide. For smaller values of $t$, on the other hand, the results of the two-dimensional waveguide deviate from those of the three-dimensional waveguide. The value of $|\phi|$ as a function of YIG, Bi:YIG, or Bi:GdIG film thickness is shown in Fig. 3(b). The value of $|\phi|$ becomes larger with an increase of the Faraday rotation coefficient $\delta$. Chen and Kumarswami [3] have reported that the guided layer thickness to give the maximum nonreciprocal phase 


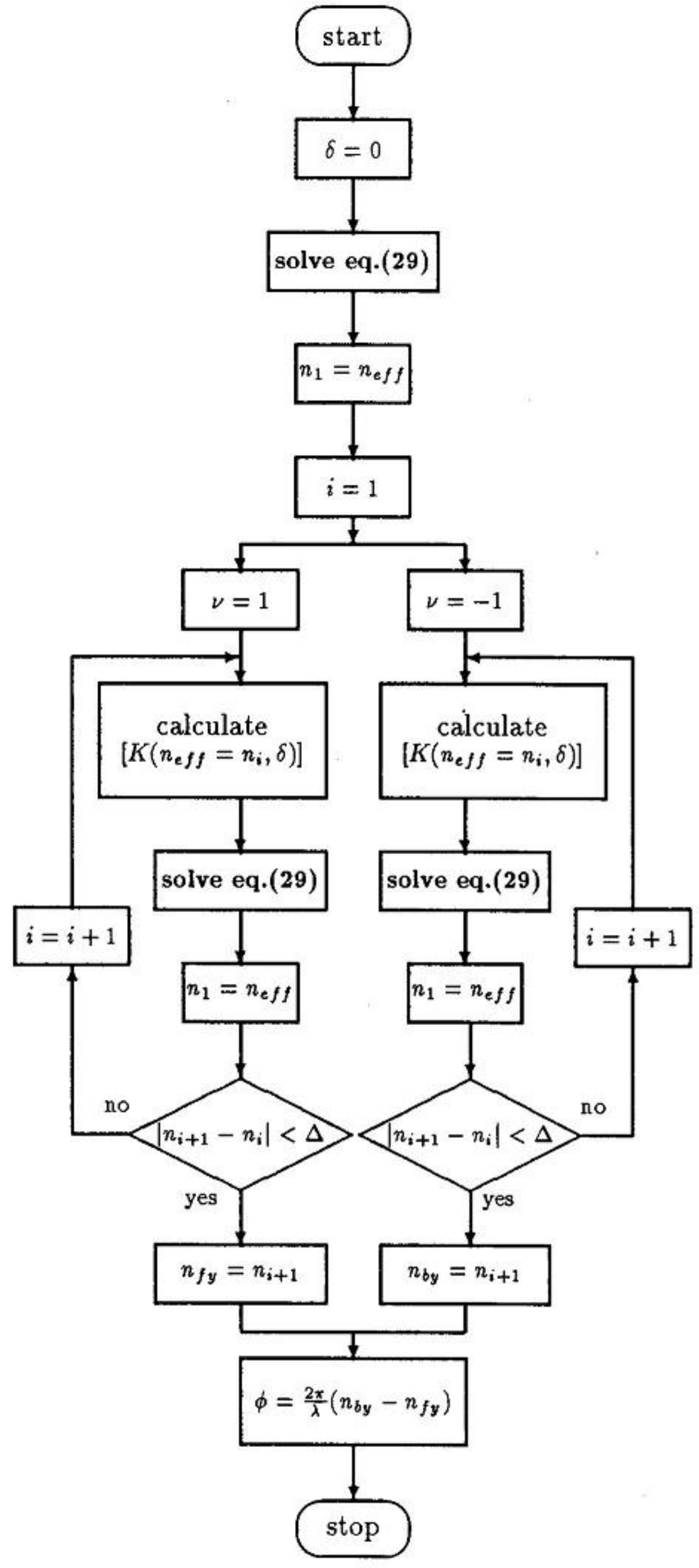

Fig. 2. Calculation of the $E^{y}$ modes.

TABLE I

Magnetooptic Material Constants

\begin{tabular}{lll}
\hline Material & $n$ & $\delta$ \\
LaGa:YIG & 2.18 & $3.2 \times 10^{-4}$ \\
YIG & 2.18 & $3.4 \times 10^{-4}$ \\
Bi:YIG & 2.25 & $-8.9 \times 10^{-4}$ \\
Bi:GdIG & 2.40 & $-4.3 \times 10^{-3}$ \\
Ce:YIG & 2.23 & -0.019 \\
\hline
\end{tabular}

shift for planar magnetooptic waveguides is approximated by $k_{0} t \sqrt{n^{2}-n_{s}^{2}} \simeq 2.2$. Using this relation, the values of $t$ become $0.41 \mu \mathrm{m}, 0.36 \mu \mathrm{m}$, and $0.29 \mu \mathrm{m}$ for YIG, Bi:YIG, and Bi:GdIG, respectively. These values approximately coincide with those in Fig. 3(b).

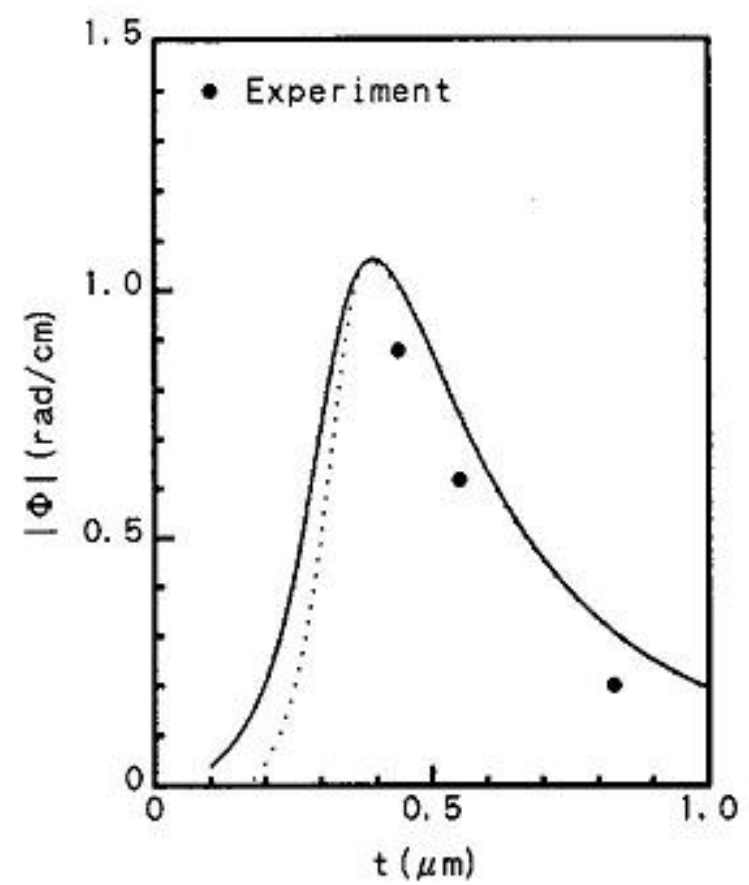

(a)

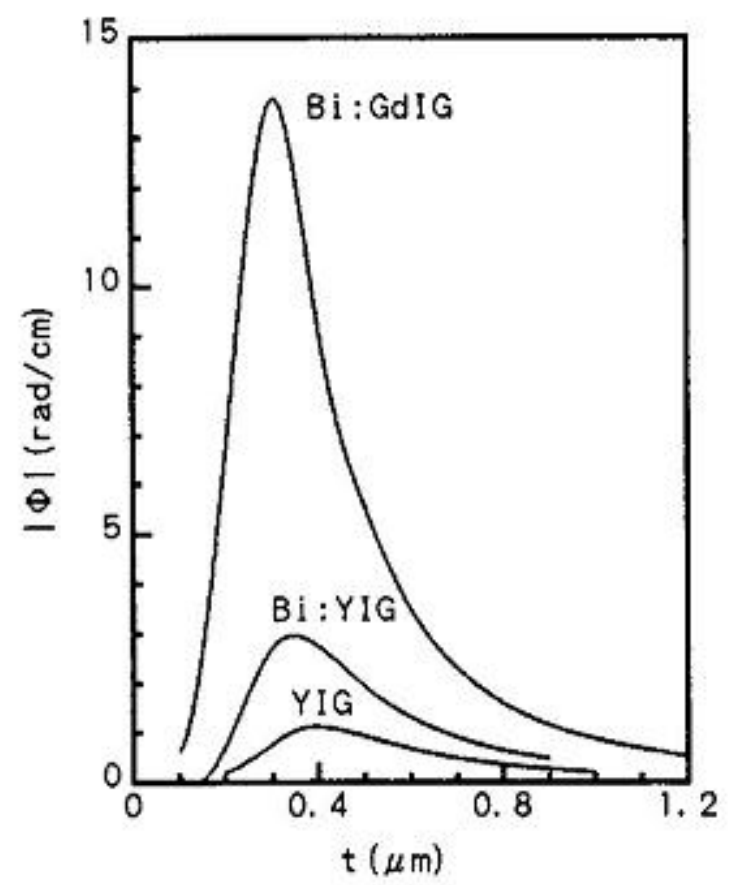

(b)

Fig. 3. Nonreciprocal phase shift of a magnetooptic rib waveguide as a function of guided layer thickness. (a) LaGa:YIG film. (b) YIG, Bi:YIG, or Bi:GdIG film.

The magnitude of the nonreciprocal phase shift of a rib waveguide on a YIG substrate as a function of guided layer thickness is shown in Fig. 4(a). The difference between the refractive indexes of a YIG film and an isotropic substrate in Fig. 3(b) is 0.23 . The same index difference is obtained for $n_{f}=2.41$ in Fig. 4(a). Comparing these two cases, we can find that the nonreciprocal phase shift becomes larger for the structure using a magnetooptic material as a substrate. Furthermore, the nonreciprocal phase shift becomes larger with an increase of the refractive index difference between a guided layer and a substrate. For a rib waveguide with a guided layer of $n_{f}=3.44$ on a YIG, Bi:YIG, or Bi:GdIG substrate, the nonreciprocal phase shift as a function of guided layer thickness is shown in Fig. 4(b). A larger value of $|\phi|$ is obtained for a Bi:GdIG substrate.

\section{B. Magnetooptic Ridge Waveguides}

We consider the magnetooptic ridge waveguides as shown in Fig. 1(c) and (d), where $W=3 \mu \mathrm{m}, d=20 \mathrm{~nm}, X=20.6 \mu \mathrm{m}$, $Y_{s}=1.7 \mu \mathrm{m}$, and $Y_{c}=0.5 \mu \mathrm{m}$. The guided layer thickness $t$ is optimized so as to give the maximum nonreciprocal phase shift in case of a rib waveguide. Isotropic and magnetooptic materials are used as a high refractive index loading layer 


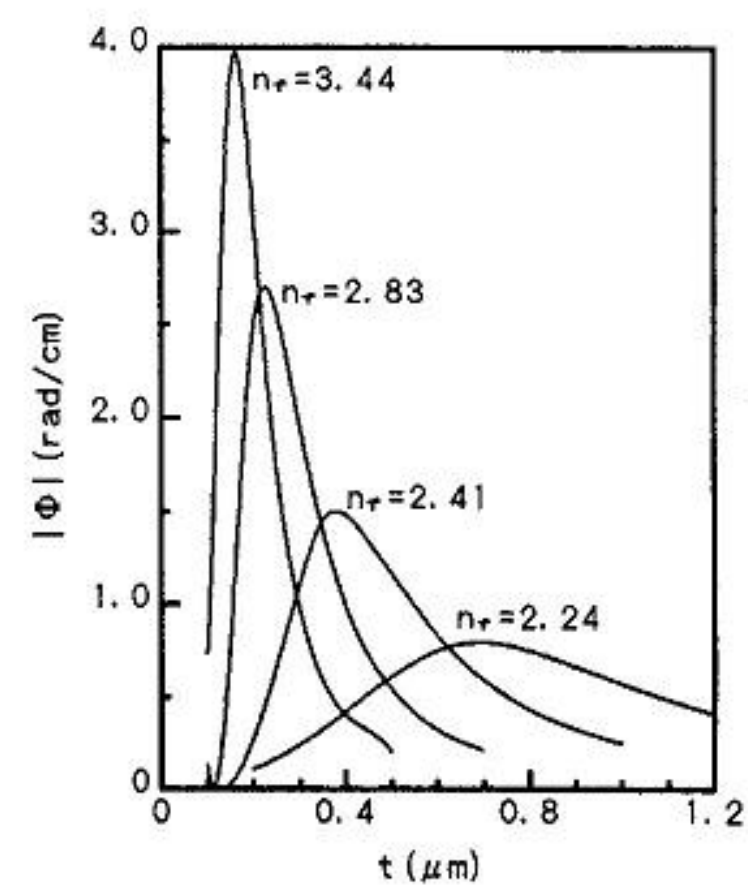

(a)

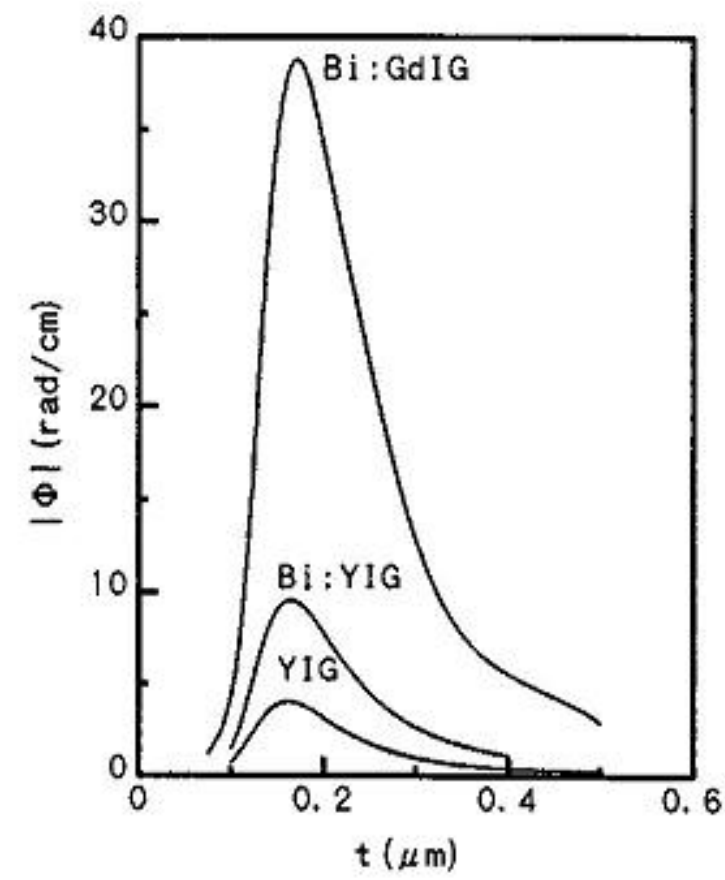

(b)

Fig. 4. Nonreciprocal phase shift of a rib waveguide on a magnetooptic substrate as a function of guided layer thickness. (a) YIG substrate. (b) YIG, Bi:YIG, or Bi:GdIG substrate.

in Fig. 1(c) and (d), respectively. For a ridge waveguide with a LaGa:YIG film of $t=0.44 \mu \mathrm{m}$ [7], the magnitude of the nonreciprocal phase shift as a function of isotropic loading layer thickness is shown in Fig. 5(a) by the solid line, where $n_{f}=2.60$. The results obtained agree approximately with the experimental results [7]. The results of the planar waveguide $(d=0)$ are also shown in Fig. 5(a), by the dotted line. Since the rib height $d$ is extremely small compared with the guided layer thickness, the ridge waveguide structure considered here is almost like the planar structures. Fig. 5(b) shows the magnitude of the nonreciprocal phase shift of a ridge waveguide with a Bi:GdIG film of $t=0.28 \mu \mathrm{m}$ as a function of isotropic loading layer thickness, where $n_{f}=2.60$ or 3.44. The value of $|\phi|$ becomes larger with an increase of the refractive index $n_{f}$ of a loading layer. While the nonreciprocal phase shift becomes larger, the change of $|\phi|$ against the loading layer thickness in the vicinity of its maximum value is very abrupt. That is to say, this structure has a small tolerance in the loading layer thickness control.

The magnitude of the nonreciprocal phase shift of a ridge waveguide with a Bi:GdIG film of $t=0.28 \mu \mathrm{m}$ as a function of magnetooptic loading layer thickness is also shown in Fig. 5(b), where a Ce:YIG film is used as a loading layer. We

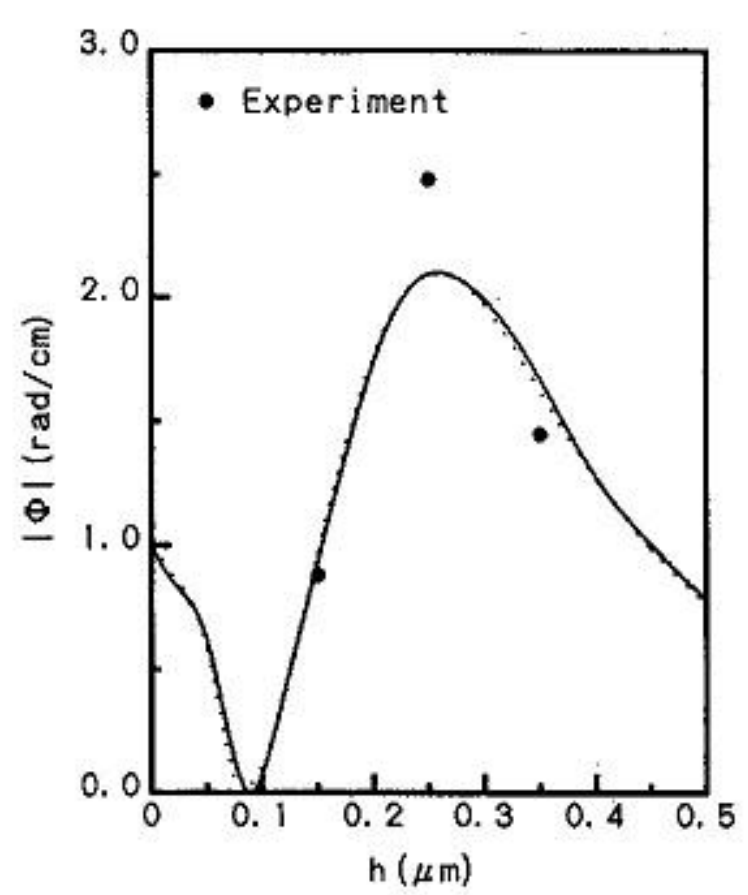

(a)

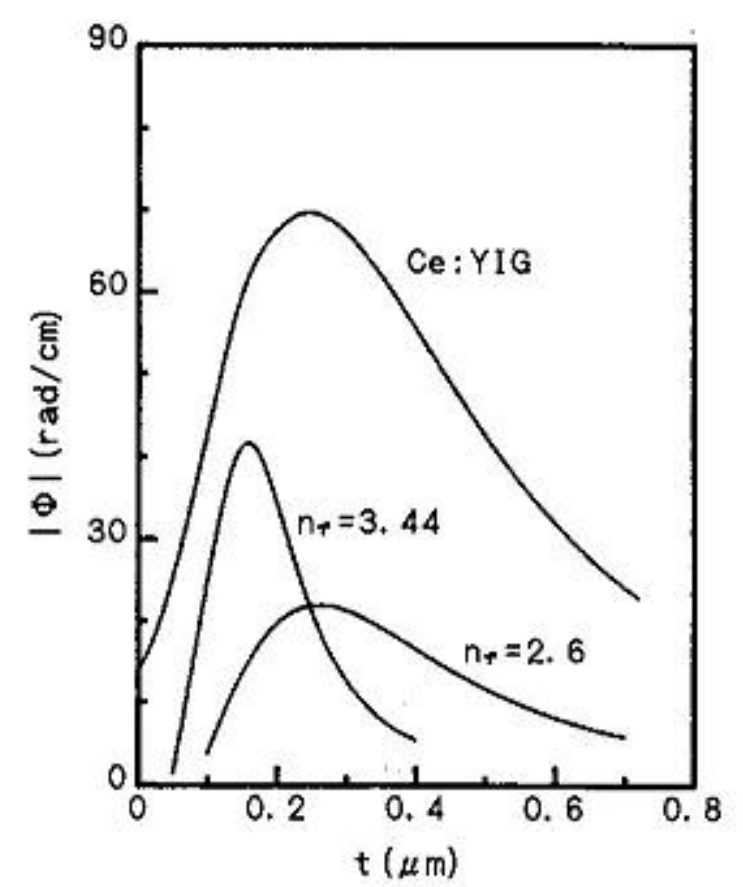

(b)

Fig. 5. Nonreciprocal phase shift of a magnetooptic ridge waveguide as a function of loading layer thickness. (a) LaGa:YIG guided layer of $t=0.44 \mu \mathrm{m}$. (b) BitGdIG guided layer of $t=0.28 \mu \mathrm{m}$.

can find that when using a magnetooptic material as a loading layer, the value of $|\phi|$ becomes larger and the change of $|\phi|$ against the loading layer thickness becomes gentler.

\section{CONCLUSIONS}

A scalar finite-element method was developed for the analysis of magnetooptic channel waveguides. In this approach, the nonphysical spurious solutions do not appear. To show the validity of this method, computed results were compared with the earlier experimental results. Also, the structures with larger nonreciprocal phase shift were investigated in detail.

The nonreciprocal phase shift is larger for an isotropic guided layer on a magnetooptic substrate than for a magnetooptic guided layer on an isotropic substrate. The larger the difference between the refractive indexes of a guided layer and a substrate becomes, the larger the nonreciprocal phase shift becomes. In the structure with an additional loading layer, the nonreciprocal phase shift is larger and its change against the loading layer thickness becomes gentler when using a magnetooptic material as a loading layer than when using an isotropic material as a loading layer.

Although a scalar finite-element approach is very convenient, its formulation is approximate in a strict sense. 
We are now working on a vector finite-element method for magnetooptic channel waveguides.

\section{REFERENCES}

[1] T. Mizumoto and Y. Naito, "Nonreciprocal propagation characteristics of YIG thin film," IEEE Trans. Microwave Theory Tech., vol. MTT-30, pp. 922-925, June 1982.

[2] T. Mizumoto, K. Oochi, T. Harada, and Y. Naito, "Measurement of optical nonreciprocal phase shift in a Bi-substituted $\mathrm{Gd}_{3} \mathrm{Fe}_{5} \mathrm{O}_{12}$ film and application to waveguide-type optical circulator," J. Lightwave Technol., vol. LT-4, pp. 347-352, Mar. 1986.

[3] C.-L. Chen and A. Kumarswami, "Nonreciprocal TM-mode thin film phase shifters," Appl. Opt., vol. 25, pp. 3664-3670, Oct. 1986.

[4] K. Matsubara and H. Yajima, "Analysis of Y-branching optical circulator using magnetooptic medium as a substrate," J. Lightwave Technol., vol. 9, pp. 1061-1067, Sept. 1991.

[5] E. A. J. Marcatili, "Dielectric rectangular waveguide and directional coupler for integrated optics," Bell Syst. Tech. J., vol. 48, pp. 2071-2102, Sept. 1969.

[6] M. Tateda and T. Kimura, "Analysis of rectangular waveguide isolator," J. Lightwave Technol., vol. LT-1, pp. 214-223, Mar. 1983.

[7] H. Inuzuka, Y. Okamura, and S. Yamamoto, "Nonreciprocal phase characteristics of single-mode magneto-optic rib waveguides," Trans. IECE Japan, vol. J71-C, pp. 702-708, May 1988 (in Japanese).

[8] H. Inuzuka, Y. Okamura, and S. Yamamoto, "Magnetooptic nonreciprocal phase shift in (YLa) ${ }_{3}(\mathrm{FeGa})_{5} \mathrm{O}_{12}$ single-mode channel waveguides," J. Appl. Phys., vol. 64, no. 3, pp. 1575-1577, Aug. 1988.

[9] M. Koshiba, K. Hayata, and M. Suzuki, "Approximate scalar finiteelement analysis of anisotropic optical waveguides," Electron. Lett., vol. 18, pp. 411-413, May 1982.

[10] , "On accuracy of approximate scalar finite-element analysis of dielectric optical waveguides," Trans. IECE Japan, vol. E66, pp. 157-158, Feb. 1983.

[11] — "Approximate scalar finite-element analysis of anisotropic optical waveguides with off-diagonal elements in a permittivity tensor," IEEE Trans. Microwave Theory Tech., vol. MTT-32, pp. 587-593, June 1984.

[12] B. M. A. Rahman, F. A. Fernandez, and J. B. Davies, "Review of finite element methods for microwave and optical waveguides," Proc. IEEE, vol. 79, pp. 1442-1448, Oct. 1991.

[13] M. Gomi, K. Satoh, and M. Abe, "Giant Faraday rotation of Cesubstituted YIG films epitaxially grown by RF sputtering," Japan. J. Appl. Phys., vol. 27, pp. L1536-L1538, Aug. 1988.
[14] Y. Miyazaki, M. Mori, and K. Akao, "Nonreciprocal and loss properties of waveguide type optical isolator using magnetooptic effects in garnet crystals," IOOC '77 Tech. Dig., P-1 (Tokyo), July 1977.

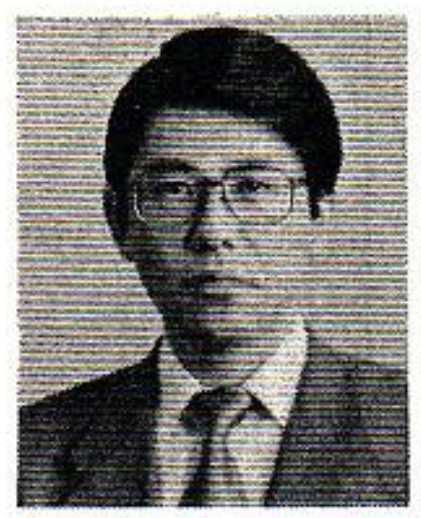

Masanori Koshiba (SM'84) was born in Sapporo, Japan, on November 23, 1948. He received the B.S., M.S., and Ph.D. degrees in electronic engineering from Hokkaido University, Sapporo, Japan, in 1971, 1973, and 1976, respectively.

In 1976, he joined the Department of Electronic Engineering, Kitami Institute of Technology, Kitami, Japan. From 1979 to 1987, he was an Associate Professor of Electronic Engineering at Hokkaido University, and in 1987 he became a Professor there. He has been engaged in research on lightwave technology, surface acoustic waves, magnetostatic waves, microwave field theory, and applications of finite-element and boundaryelement methods to field problems.

Dr. Koshiba is a member of the Institute of Electronics, Information and Communication Engineers (IEICE), the Institute of Television Engineers of Japan, the Institute of Electrical Engineers of Japan, the Japan Society for Simulation Technology, and the Japan Society for Computational Methods in Engineering. In 1987, he was awarded the 1986 Paper Award by the IEICE.

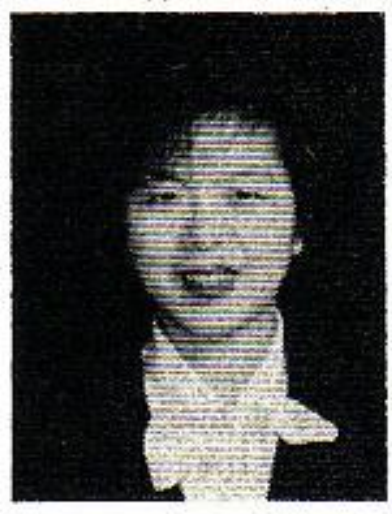

Xiu-Ping Zhuang was born in Anhui Province, China, on July 1,1963 . She received the B.S. degree in communication engineering from Guilin College of Electronic Engineering, Guilin, China, in 1986. She is presently studying toward the M.S. degree in electronic engineering at Hokkaido University, Sapporo, Japan.

Ms. Zhuang is a member of the Institute of Electronics, Information and Communication Engineers of Japan. 